\title{
EXISTENCE AND NON-EXISTENCE OF ENTIRE POSITIVE SOLUTIONS FOR QUASILINEAR SYSTEMS WITH SINGULAR AND SUPER-LINEAR TERMS
}

\author{
HONGHUI YIN AND ZUODONG YANG
}

Abstract. We establish the results concerning existence and non-existence of entire positive solutions for the nonlinear elliptic systems

$$
\left\{\begin{array}{l}
-\Delta_{p} u=a(x) u^{m}+\lambda c(x) v^{n}, \quad x \in \mathbb{R}^{N}, \\
-\Delta_{q} v=b(x) v^{l}+\theta c(x) u^{n}, \quad x \in \mathbb{R}^{N}, \\
u, v>0, x \in \mathbb{R}^{N} \text { and } u \rightarrow 0, v \rightarrow 0 \text { as }|x| \rightarrow \infty,
\end{array}\right.
$$

where $1<p, q<N$ and $\lambda, \theta \geqslant 0$ are nonnegative parameters, $a, b, c: \mathbb{R}^{N} \rightarrow[0, \infty)$ are locally Hölder continuous functions not identically zero, and $-\infty<m<p-1,-\infty<l<q-1, \max \{p-$ $1, q-1\}<n$. The main purpose of this paper is to extend the principal theorem of $\mathrm{Xu}$ and Yang in [23] which concerned single equation.

Mathematics subject classification (2010): 35B09, $35 \mathrm{~J} 47$.

Keywords and phrases: nonexistence, entire solution, sub-linear and super-linear, singular.

\section{REFERENCES}

[1] J. ALI AND R. SHIVAJI, Existence results for classes of Laplacian systems with sign-changing weight, Appl. Maths. Letters, 20 (2007), 558-562.

[2] W. Allegretto And Y. X. Huang, A picone's identity for the p-Laplacian and applications, Nonlinear Analysis, 32, 7 (1998), 819-830.

[3] A. CANADA, P. DRAVEK AND J. L. GAmEZ, Existence of positive solutions form some problems with nonlinear diffusion, Trans. Amer. Math. Soc., 349, 10 (1997), 4231-4249.

[4] F. CiRSTEA AND V. D. RADUlesCU, Entire solutions blowing up at infinity for semilinear elliptic systems, J. Math. Pures Appl., 81 (2002), 827-846.

[5] F. CiRSTEA AND V. D. RADUlESCU, Existence and uniqueness of positive solutions to a semilinear elliptic problem in $R^{N}$, J. Math. Anal. Appl., 229 (1999), 417-425.

[6] F. CiRsteA AND V. RADUlESCU, Entire solutions blowing up at infinite for semilinear elliptic systems, J. Math. Pures Appl, 81 (2002), 827-846.

[7] Ph. Clement, R. Manasevich And E. Mitidieri, Positive solutions for a quasilinear system via blow up, Comm. in Partial Diff. Eqns., 18, 12 (1993), 2071-2106.

[8] Ph. Clement, D. G. De Figueiredo And E. Mitidieri, Positive solutions of semilinear elliptic systems, Comm. in Partial Diff. Eqns., 17, 5/6 (1992), 923-940.

[9] R. EsteBAN AND J. L. VÁSQUEZ, On the equation of turbulent filtration in one-dimensional porous media, Nonlinear Analysis, 10 (1986), 1303-1325.

[10] P. L. Felmer, R. MANASEvich And F. DE Thelin, Existence and uniqueness of positive solutions for certain quasilinear elliptic system, Comm. in Partial Diff. Eqns., 17 (1992), 2013-2029.

[11] W. FENG AND X. LIU, Existence of entire solution of a singular semilinear elliptic problem, Acta Math. Sin., Engl. Ser., 20, 6 (2004), 983-988.

[12] Z. M. GuO, Existence of the positive radial solutions for certain of quasilinear elliptic systems, Chin. Ann. Math., Ser. A, 17, 5 (1996), 573-582. 
[13] Z. M. Guo, Some existence and multiplicity results for a class of quasilinear elliptic equations, Nonlinear Anal., 18, 10 (1992), 957-971.

[14] Z. M. Guo, Existence of positive radial solutions for a class of quasilinear elliptic systems in annular domains, Chinese Journal of Contemporary Math., 17, 4 (1996), 337-350.

[15] A. V. LAIR, Large solutions of mixed sublinear/superlinear elliptic equations, J. Math. Anal. Appl., 346 (2008), 99-106.

[16] A. V. LAIR, A. W. ShaKer, Entire solutions of a singular elliptic problem, J. Math. Anal. Appl., 200 (1996), 498-505.

[17] A. V. LAIR, A. W. SHAKER, Classical and weak solutions of a singular semi-linear elliptic problem, J. Math. Anal. Appl., 211 (1997), 371-385.

[18] A. V. LAIR AND A. W. WOOD, Existence of entire large positive solutions of semilinear elliptic systems, J. Diff Equations, 164 (2000), 380-394.

[19] V. MikLJUKov, On the asymptotic properties of sub-solutions of quasilinear equations of elliptic type and mappings with bounded distortion, Sbornik Mathematics (N.S.) 111 (1980), (Russian).

[20] E. MitIDIERI, Nonexistence of positive solutions of semilinear elliptic system in $\mathbb{R}^{N}$, Diff. Integral Equations, 9 (1996), 465-479.

[21] C. A. SANTOS, On Ground state solutions for singular and semi-linear problems including superlinear terms at the infinite, Nonlinear Anal., 71 (2009), 6038-6043.

[22] K. UHLEMBECK, Regularity for a class of nonlinear elliptic systems, Acta Mathematica, 138 (1977), $219-240$.

[23] B. XU AND Z. D. YANG, Entire bounded solutions for a class of quasilinear elliptic equations, Boundary Value Problems 2007. Art. ID 16407, 1-8.

[24] Z. D. YANG AND Q. S. LU, Non-existence of positive radial solutions for a class of quasilinear elliptic system, Comm. Nonlinear Sci. Numer. Simul., 5, 4 (2000), 184-187.

[25] Z. D. YANG AND Q. S. LU, Nonexistence of positive solutions to a quasilinear elliptic system and blow-up estimates for a quasilinear reaction-diffusion system, J. Computational and Appl. Math., 150 (2003), 37-56.

[26] C. YARUR, Existence of continuous and singular ground states for semilinear elliptic systems, Electron. J. Differential Equations, 1 (1998), 1-27.

[27] D. Ye AND F. ZHOU, Invariant criteria for existence of bounded positive solutions, Discrete and Continuous Dynamical Systems, 12, 3 (2005), 413-424.

[28] H. H. YIN AND Z. D. YANG, New results on the existence of bounded positive entire solutions for quasilinear elliptic systems, Appl. Math. Comput, 190 (2007), 441-448; 177 (2006), 606-613.

[29] Z. ZHANG, A remark on the existence of entire solutions of a singular semi-linear elliptic problem, J. Math. Anal. Appl., 215 (1997), 570-582. 